\title{
SERUM SODIUM AND POTASSIUM PROFILE IN ADULT HEAD INJURY PATIENTS AND ITS EFFECT ON FINAL OUTCOME
} Sushanta Kumar Das ${ }^{1}$, Paresh Kumar Gouda², Niranjan Sahoo 3 , Bhupati Bhusan Das ${ }^{4}$, Sanjeeb Kumar Pradhan ${ }^{5}$, Anoob Mohamed 6 ,
Nrusingh Charan Dash ${ }^{7}$, Pathi Ravi Teja

1 Professor, Department of General Surgery, MKCG Medical College, Berhampur.

${ }^{2}$ Resident, Department of General Surgery, MKCG Medical College, Berhampur.

${ }^{3}$ Assistant Professor, Department of General Surgery, MKCG Medical College, Berhampur.

${ }^{4}$ Assistant Professor, Department of General Surgery, MKCG Medical College, Berhampur.

${ }_{5}^{5}$ Senior Resident, Department of General Surgery, MKCG Medical College, Berhampur.

${ }^{6}$ Resident, Department of General Surgery, MKCG Medical College, Berhampur.

${ }^{7}$ Resident, Department of General Surgery, MKCG Medical College, Berhampur.

${ }^{8}$ Resident, Department of General Surgery, MKCG Medical College, Berhampur.

\section{ABSTRACT}

\section{BACKGROUND}

Objective- To study the incidence of abnormalities of serum $\mathrm{Na}^{+}$and $\mathrm{K}^{+}$in all adult head injury patients and to analyse its effect on management and final outcome.

\section{MATERIALS AND METHODS}

A prospective study was done in the patients of head injury admitted to the surgical wards of MKCG Medical College \& Hospital, Berhampur $2 \frac{1}{2} 2$ years from August 2014.

\section{RESULTS}

Out of 340 patients taken, $17.35 \%$ had isolated hyponatraemia, $11.18 \%$ had hypernatraemia, $12.35 \%$ had hypokalaemia $\& 5 \%$ had hyperkalaemia. All those abnormalities were detected within first week of admission. Majority of the cases had low Glasgow Coma Score ( $\leq 8)$. Incidence of Subarachnoid Haemorrhage (SAH) was high in the hyponatraemic patients. All the patients of hyponatraemia had Syndrome of Inappropriate ADH secretion (SIADH). Amongst hypernatraemic patients, incidence of diabetes insipidus was $15.79 \%$. Resolution of hyponatraemia, hypernatraemia, hypokalaemia and hyperkalaemia took 1.92, 2.70, 1.71 and 1.82 days respectively. Length of hospital stay (LOS) in patients with hyponatraemia, hypernatraemia, hypokalaemia and hyperkalaemia was $7.4,8.8,6.3,7.1$ days respectively. In rest of the patients, the average LOS was 4.4 days. Mortality rate is highest $(28.95 \%)$ in hypernatraemic patients. Hypokalaemia occurred first and resolved quickly. Hypernatraemia took a little longer to resolve.

\section{CONCLUSION}

Hyponatraemia is the most common electrolyte abnormality in head injury patients. Syndrome of Inappropriate ADH secretion (SIADH) may be the main cause of hyponatraemia following head injury. Electrolyte imbalance occurs mostly in the first week following trauma and takes around 72 hours for resolution of imbalance. Morbidity \& Mortality rate is higher as compared to patients without electrolyte imbalance.

\section{KEYWORDS}

Head Injury, Hyponatraemia, Hypernatraemia, Hypokalaemia, Hyperkalaemia, Glasgow Coma Scale, Glasgow Outcome Scale, Syndrome of Inappropriate ADH Secretion, Cerebral Salt Wasting Syndrome, Diabetes Insipidus.

HOW TO CITE THIS ARTICLE: Das SK, Gouda PK, Sahoo N, et al. Serum sodium and potassium profile in adult head injury patients and its effect on final outcome. J. Evolution Med. Dent. Sci. 2017;6(31):2528-2532, DOI: 10.14260/Jemds/2017/547

\section{BACKGROUND}

Electrolyte imbalance after head injury is of common occurrence. Apart from primary injuries sustained during trauma, the secondary injuries in the post-traumatic phase like hypovolaemia, electrolyte imbalance, hyperosmolality,

Financial or Other, Competing Interest: None.

Submission 12-03-2017, Peer Review 06-04-2017,

Acceptance 11-04-2017, Published 17-04-2017.

Corresponding Author:

Dr. Paresh Kumar Gouda,

C/o. Profess. S. K. Das,

$P G$ Department of General Surgery,

MKCG Medical College,

Berhampur-760004, Odisha.

E-mail: pareshons@gmail.com

DOI: $10.14260 /$ jemds $/ 2017 / 547$

(c) (i) etc., hold importance defining the outcome of the patient in the form of morbidity or mortality. ${ }^{1}$ Amongst all the cations and anions, sodium $\left(\mathrm{Na}^{+}\right)$and potassium $\left(\mathrm{K}^{+}\right)$are more important in the regulation of intracranial homeostasis.

\begin{abstract}
Aim
To study the incidence of abnormalities of serum $\mathrm{Na}^{+}$and $\mathrm{K}^{+}$ in all adult head injury patients and to analyse its effect on management and final outcome in these patients.
\end{abstract}

\section{MATERIALS \& METHODS}

The patients admitted to surgical wards of MKCG Medical College and Hospital, Berhampur with head injury (without polytrauma) for a period of $2 \frac{1}{2}$ years from August 2014, consecutively from both sexes and $>14$ years of age were included in the study. After initial assessment and CT scan of 
brain and treatment in emergency, patients were evaluated with other routine investigations. Meanwhile, all the relevant history was taken from the patients and/or relatives about previous comorbid illnesses and history of any drug intake.

As this study was all about electrolyte imbalance and primarily serum $\mathrm{Na}^{+}$and $\mathrm{K}^{+}$, the serum levels of both the components were checked regularly. The estimation of serum sodium and potassium was done in an electrolyte analyser machine (iSENS ${ }^{\circledR}$, model- iSmart 30) which uses Ion Sodium Electrodes method to detect serum electrolytes. Hyponatraemia $(<135 \mathrm{mEq} / \mathrm{L})$, hypernatraemia $(>145$ $\mathrm{mEq} / \mathrm{L})$, hypokalaemia $(<3.6 \mathrm{mEq} / \mathrm{L})$, hyperkalaemia $(>5.5$ $\mathrm{mEq} / \mathrm{L}$ ) were assessed, noted and analysed. Serum levels of sodium and potassium were assessed once every day till the discharge/death of the patient. Any abnormality when detected was noted. Fluid intake and urine output were measured every day and specific gravity was measured twice daily by dipstick method using rapid urine analysis kit (URISTIK $^{\circledR}$ 10D urine analysis strips). It helped to differentiate between cause of hyponatraemia which could be due to Syndrome of Inappropriate ADH secretion (SIADH) (reduction in urine volume as compared to previous day fluid intake) and Cerebral Salt Wasting Syndrome (CSWS) (polyuric state and hyponatraemia) and treated accordingly. Suspected cases of SIADH were treated with fluid restriction only. There were no cases of CSWS in this study.

Hypernatraemia with decreased urine volume and urine specific gravity indicated towards hypovolaemia due to blood loss and treated with fluid resuscitation, but hypernatraemia with increased urine volume with iso-osmolar urine indicated diabetes insipidus and treated with fluid replacement only with close observation. All the cases responded well to this method, and treatment with aqueous vasopressin or desmopressin was not needed.

Potassium abnormalities were detected in many cases. These cases were monitored closely with serum values and electrocardiogram as it could cause cardiac arrhythmias and sudden deterioration of the patients. With regular followup, the improvement in patients' general condition and the final outcome of the patients were noted in the form of Glasgow Outcome Scale Score.

\section{Inclusion Criteria}

All adult patients (15 years of age and above) with closed head injury.

\section{Exclusion Criteria}

1. Patients with multiple types of electrolyte abnormalities either synchronously or one after another as it might hinder the evaluation as this study was to find the effect of a single type of electrolyte abnormality on outcome.

2. Patients with polytrauma.

3. Patients not willing to take part in the study.

4. Patients taking diuretics.

5. Patients with a known history of Diabetes Insipidus.

6. Patients with Diabetes Mellitus with or without insulin therapy.

7. Patients with renal, hepatic and adrenal diseases.

8. Patients with mineralocorticoid treatment.

9. Patients with multiple types of lesion in NCCT of brain as the association of electrolyte imbalance with specific type of lesion was also a part of the study. So patients with single type of lesions were included.

\section{RESULTS}

Incidence of Serum Electrolyte Imbalance

Incidence of hyponatraemia was comparatively more than other abnormalities.

\begin{tabular}{|c|c|c|}
\hline Electrolyte Imbalance & No. of Cases & Percentage (\%) \\
\hline Hyponatraemia & 59 & 17.35 \\
\hline Hypernatraemia & 38 & 11.18 \\
\hline Hypokalaemia & 42 & 12.35 \\
\hline Hyperkalaemia & 17 & 5 \\
\hline Rest of the cases & 184 & 54.12 \\
\hline \multicolumn{2}{|c|}{ Table 1. Incidence of Serum Electrolyte Imbalance } \\
\hline
\end{tabular}

Day of Detection of Electrolyte Abnormality after Admission

54 out of 59 cases of hyponatraemia were detected within first 4 days (91.51\%) with peak incidence within 24-72 hours from the time of admission. In cases of hypernatraemia, 33 out of 38 cases occurred within $2^{\text {nd }}$ to $5^{\text {th }}$ day (86.85\%) from injury. Hypokalaemia occurred within first 96 hours from injury in 38 out 42 cases $(92.86 \%)$. All cases of hyperkalaemia occurred within the first 3 days.

\begin{tabular}{|c|c|c|c|c|}
\hline 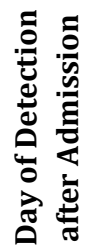 & 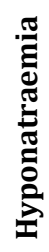 & 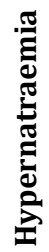 & 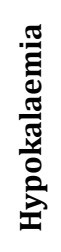 & 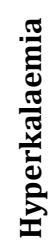 \\
\hline 1 & 9 & 2 & 5 & 10 \\
\hline 2 & 23 & 4 & 12 & 6 \\
\hline 3 & 15 & 12 & 13 & 1 \\
\hline 4 & 7 & 13 & 8 & 0 \\
\hline 5 & 3 & 4 & 3 & 0 \\
\hline 6 & 1 & 2 & 1 & 0 \\
\hline 7 & 1 & 1 & 0 & 0 \\
\hline Total & 59 & 38 & 42 & 17 \\
\hline
\end{tabular}

Relationship of Sodium \& Potassium Abnormalities with Initial GCS Score

In all types of electrolyte imbalance, initial GCS Score was low. 29 out of 59 (49.15\%) hyponatraemic patients, 24 out of $38(63.16 \%)$ hypernatraemic patients, 18 out of $42(42.86 \%)$ hypokalaemic patients and 9 out of 17 (52.94\%) hyperkalaemic patients were severely injured and had GCS Score $\leq 8$.

\begin{tabular}{|c|c|c|c|}
\hline $\begin{array}{c}\text { Electrolyte } \\
\text { Imbalance }\end{array}$ & $\begin{array}{c}\text { GCS } \\
\mathbf{( 3 - 8 )}\end{array}$ & $\begin{array}{c}\text { GCS } \\
\mathbf{( 9 - 1 2 )}\end{array}$ & $\begin{array}{c}\text { GCS } \\
\mathbf{( 1 3 - 1 5 )}\end{array}$ \\
\hline Hyponatraemia & 29 & 17 & 13 \\
\hline Hypernatraemia & 24 & 7 & 7 \\
\hline Hypokalaemia & 18 & 13 & 11 \\
\hline Hyperkalaemia & 9 & 3 & 5 \\
\hline Total & $\mathbf{8 3}$ & $\mathbf{4 0}$ & $\mathbf{3 5}$ \\
\hline \multicolumn{3}{|c|}{ Table 3. Relationship of Sodium \& Potassium } \\
Abnormalities with Initial GCS Score \\
\hline
\end{tabular}




\begin{tabular}{|c|c|c|c|c|c|c|c|c|}
\hline Electrolyte Abnormality & EDH & SDH & SAH & IVH & DAI & $\begin{array}{c}\text { Brain } \\
\text { Contusion }\end{array}$ & Skull Fracture & Total \\
\hline Hyponatraemia & 8 & 6 & 10 & 2 & 5 & 15 & 13 & 59 \\
\hline Hypernatraemia & 7 & 5 & 4 & 1 & 3 & 10 & 8 & 38 \\
\hline Hypokalaemia & 5 & 4 & 5 & 2 & 2 & 13 & 11 & 42 \\
\hline Hyperkalaemia & 3 & 3 & 2 & 0 & 1 & 3 & 5 & 17 \\
\hline Rest of the cases & 24 & 18 & 16 & 9 & 10 & 46 & 61 & 184 \\
\hline Total & $\mathbf{4 7}$ & $\mathbf{3 6}$ & $\mathbf{3 7}$ & $\mathbf{1 4}$ & $\mathbf{2 1}$ & $\mathbf{8 7}$ & $\mathbf{9 8}$ & $\mathbf{3 4 0}$ \\
\hline \multicolumn{2}{r}{} \\
\hline
\end{tabular}

Relationship between Urine Specific Gravity (USP) and Electrolyte Abnormality

A total of $44(74.58 \%)$ patients with hyponatraemia had decreased urine volume and increased specific gravity and rest of the patients had unequivocal reading. All these cases might have SIADH. Amongst hypernatraemic patients 22 $(57.90 \%)$ had hyperosmolar urine and cause may be due to hypovolemia and 6 (15.79\%) had hypo-osmolar urine and may have diabetes insipidus.

\begin{tabular}{|c|c|c|c|c|}
\hline \multirow[b]{2}{*}{$\begin{array}{c}\text { Electrolyte } \\
\text { Abnormality }\end{array}$} & \multicolumn{3}{|c|}{ Urine } & \multirow[b]{2}{*}{ Total } \\
\hline & $\begin{array}{c}\text { Hypo- } \\
\text { Osmolar } \\
\text { USP } \\
<1.005\end{array}$ & $\begin{array}{c}\text { Iso- } \\
\text { Osmolar } \\
\text { USP } \\
1.005- \\
1.015 \\
\end{array}$ & $\begin{array}{c}\text { Hyper- } \\
\text { Osmolar } \\
\text { USP } \\
>\mathbf{1 . 0 1 5}\end{array}$ & \\
\hline Hyponatraemia & 0 & 15 & 44 & 59 \\
\hline Hypernatraemia & 6 & 10 & 22 & 38 \\
\hline Hypokalaemia & 10 & 17 & 15 & 42 \\
\hline Hyperkalaemia & 2 & 5 & 12 & 17 \\
\hline Rest of cases & 22 & 98 & 64 & 184 \\
\hline
\end{tabular}

Table 5. Relationship between Urine Specific Gravity (USP) and Electrolyte Abnormality

Average Time in Days taken for Resolution of Electrolyte Imbalance with Standard Treatment

With appropriate therapy abnormalities of sodium and potassium took around 72 hours for resolution on an average.

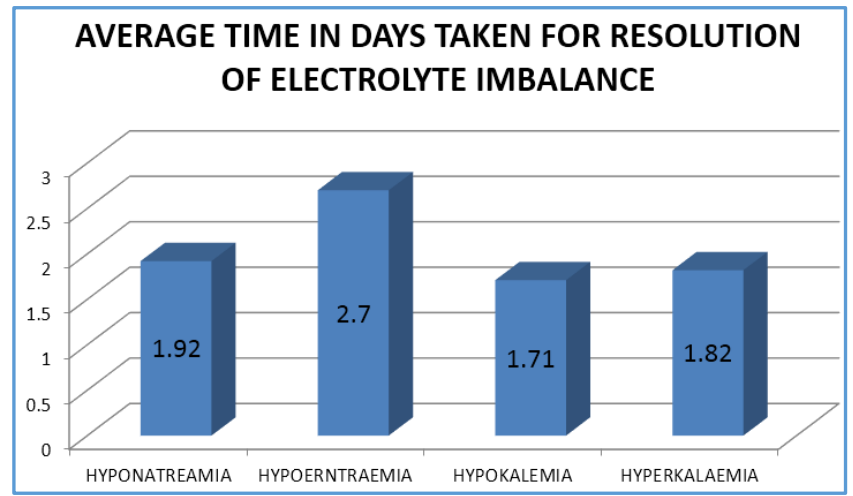

Figure 1. Average time in Days taken for Resolution of Electrolyte Imbalance with Standard Treatment Relationship of Average Length of Stay (LOS) In Hospital till Discharge and Electrolyte Abnormality (Indicator of Morbidity)

As a measure of morbidity, average length of stay (LOS) was more in all types of electrolyte imbalance as compared to patients without any electrolyte imbalance.

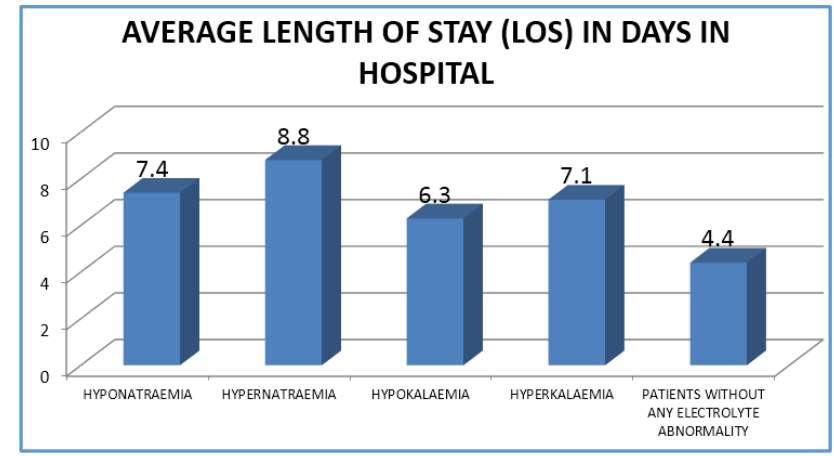

Figure 2. Relationship of Average Length of Stay (Los) in Hospital till Discharge and Electrolyte Abnormality (Indicator of Morbidity)

Mortality Rate in Electrolyte Abnormalities

Mortality rate in all the types of electrolyte imbalance in head injury was high as compared to cases without any electrolyte imbalance. Mortality rate is highest in hypernatraemic patients being 11 out of $38(28.95 \%)$ in this study.

\begin{tabular}{|c|c|c|c|}
\hline Condition & $\begin{array}{c}\text { Total No. of } \\
\text { Patients }\end{array}$ & $\begin{array}{c}\text { No. of } \\
\text { Deaths }\end{array}$ & $\begin{array}{c}\text { Mortality } \\
\text { Rate }\end{array}$ \\
\hline Hyponatraemia & 59 & 12 & $20.35 \%$ \\
\hline Hypernatraemia & 38 & 11 & $28.95 \%$ \\
\hline Hypokalaemia & 42 & 7 & $16.67 \%$ \\
\hline Hyperkalaemia & 17 & 4 & $23.53 \%$ \\
\hline Rest of the cases & 184 & 23 & $12.50 \%$ \\
\hline Total & $\mathbf{3 4 0}$ & $\mathbf{5 7}$ & $\mathbf{1 6 . 7 6 \%}$ \\
\hline
\end{tabular}

Table 6. Mortality Rate in Electrolyte Abnormalities

\section{DISCUSSION}

The incidence of hyponatraemia was found to be $17.35 \%$ which was similar to the incidence of $16.8 \%$ as stated by Nobuhiro Moro et al. ${ }^{2}$ The incidence of hypernatraemia was $11.18 \%$, but in severely injured patients with GCS Score of 38 , the incidence is 24 out of 38 i.e. $63.15 \%$ which was high as compared to study done by Umberto Maggiore et al (51.5\%) and study by Jawad Rochdi et al. (30.23\%). This may be due to hypovolaemia as much of the cases had hyperosmolar urine with high urine specific gravity (57.9\%).3, 4 The incidence of hypokalaemia was $12.35 \%$ which was less as compared to study done by Beal AL et al (27.5\%). ${ }^{5}$ The incidence of hyperkalaemia was found to be $5 \%$ of 340 patients.

In most of the cases, hyponatraemia was detected within first 4 days (91.51\%) with peak incidence within 24-72 hours from the time of admission. This was similar to the study done by S. Lohani et al, which states that hyponatraemia was detected in the first week from day of injury in majority of cases. ${ }^{6}$ In cases of hypernatraemia, most cases occurred 
within $2^{\text {nd }}$ to $5^{\text {th }}$ day $(86.85 \%)$ from injury which was also similar to the study done by Umberto Maggiore et al. ${ }^{3}$ Hypokalaemia occurred within first 96 hours from injury in majority of cases $(92.86 \%)$. Xing Wu et al also detected in their studies the majority of cases to be within 24-96 hours from injury. ${ }^{7}$ Hyperkalaemia occurred within the first 3 days in all of the cases.

In all types of electrolyte imbalance, initial GCS Score was $\leq 8.49 .15 \%$ hyponatraemic patients, $63.16 \%$ hypernatraemic patients, $42.86 \%$ hypokalaemic patients and $52.94 \%$ hyperkalaemic patients were severely injured and had GCS Score between 3-8. The result was at par with studies done by Nobuhiro Moro et al, Jawad Rochdi et al, Beal AL et al and Mirza Faisal Ahmed Rafiq et al respectively.2,4,5,8

In a study by Sherlock $M$ et al, it has been shown that hyponatraemia is common following SAH. ${ }^{9}$ In the present study, association of SAH and hyponatraemia is evident as 10 out of 59 hyponatraemic patients $(16.95 \%)$ as compared to the patients were without any electrolyte abnormalities where 16 cases out of 184 patients ( $8.7 \%$ ) had SAH.

A total of $74.58 \%$ patients with hyponatraemia had decreased urine volume and increased specific gravity whereas rest of the patients had unequivocal reading. All these cases may have had SIADH. According to a study by S. Lohani et al, it was $55.56 \% .^{6}$ In a study by Sepehri et al, all the patients $(100 \%)$ with hyponatraemia had SIADH and none of the patients had cerebral salt wasting syndrome (CSWS) signified by increased urine output. ${ }^{10}$

Amongst hypernatraemic patients, 57.90\% had hyperosmolar urine and cause may be hypovolaemia and $15.79 \%$ had hypo-osmolar urine and had diabetes insipidus. In their study, Umberto Maggiore et al found 19.2\% patients had diabetes insipidus out of all the hypernatraemic head injury patients. ${ }^{3}$

On an average, days taken for resolution of hyponatraemia with standard therapy was 1.92 days. Compared to the study by S. Lohani et al, (1.78 days) it was a little more. ${ }^{6}$ In hypernatraemic patients, the average time required for resolution was 2.70 days in an average, which was a little more than the study done by Kolmodin et al which stated that with adequate therapy, majority of cases of hypernatraemia with post-traumatic brain injury resolve within 24 to 48 hours. ${ }^{11}$ In hypokalaemic patients, the average time required for the serum potassium to be normal was 1.71 days and in hyperkalaemic patients, it was 1.82 days. As per Schaefer et al, admission hypokalaemia is more frequent in patients with head injury and resolution occurs quickly due to shifting of potassium into intracellular compartment. 12

As a measure of morbidity, average length of stay (LOS) was more in all types of electrolyte imbalance as compared to patients without any electrolyte imbalance. According to Beal $\mathrm{AL}$ et al, LOS in head injury with hypokalaemia was 8.5 days as compared to 5.6 days in other patients. ${ }^{5}$ In this study, in hypokalaemic patients, LOS was 6.3 days as compared to 4.4 days in patients without electrolyte imbalance.

Mortality rate in all the types of electrolyte imbalance in head injury was high as compared to cases without any electrolyte imbalance. Mortality rate is highest in hypernatraemic patients being $28.95 \%$ in this study. But it was less as compared to the study done by Jawad Rochdi et al who had a death rate of $76.92 \%$. It may be due to the fact that he took patients only with severe head injuries. ${ }^{4}$

\section{CONCLUSION}

Hyponatraemia is the most common electrolyte abnormality in head injury patients. SIADH is the main cause of hyponatraemia following head injury and CSWS is very rare. All types of electrolyte imbalance are seen more in severely injured patients with GCS Score of 3-8. Hyponatraemia after head injury occurs mainly due to hypovolaemia due to blood loss or use of osmotic diuretics. Hypernatraemia due to diabetes insipidus may occur but less in incidence.

Electrolyte abnormalities are usually detected in the first week following head injury. Resolution of imbalance takes 2 to 3 days in majority of cases. Hypokalaemia occurs first and resolves quickly. Hypernatraemia takes a little longer to resolve.

Morbidity is increased in all cases of head injury with electrolyte imbalance as indicated by average length of stay (LOS). Mortality rate in all the types of electrolyte imbalance in head injury was high as compared to cases without any electrolyte imbalance. Mortality rate is highest in hypernatraemic patients.

Hence, electrolyte imbalance in head injury patients is an important issue and should be taken care of as soon as evident to decrease morbidity and mortality. It should not be ignored which will lead to poor outcome.

\section{REFERENCES}

[1] Zink BJ. Traumatic brain injury. Emerg Med Clin North Am 1996;14(1):115-50.

[2] Moro N, Katayama Y, Igarashi T, et al. Hyponatremia in patients with traumatic brain injury: incidence, mechanism and response to sodium supplementation or retention therapy with hydrocortisone. Surgical Neurology 2007;68(4):387-93.

[3] Maggiore U, Picetti E, Antonucci E, et al. The relation between the incidence of hypernatremia and mortality in patients with severe traumatic brain injury. Crit Care 2009;13(4):R110.

[4] Rochdi J. Hypernatremia in patients with traumatic brain injury: about 43 cases. Research 2015;2:1463.

[5] Beal AL, Scheltema KE, Beilman GJ, et al. Hypokalemia following trauma. Shock 2002;18(2):107-10.

[6] Lohani S, Devkota UP. Hyponatremia in patients with traumatic brain injury: etiology, incidence and severity correlation. World Neurosurg 2011;76(34):355-60.

[7] $\mathrm{Wu} \mathrm{X}, \mathrm{Lu} \mathrm{X}, \mathrm{Lu} X$, et al. Prevalence of severe hypokalaemia in patients with traumatic brain injury. Injury 2015;46(1):35-41.

[8] Rafiq MFA, Ahmed N, Khan AA. Serum Electrolyte Derangements in Patients with Traumatic Brain Injury. J Ayub Med Coll Abbottabad 2013;25(1-2): 162-4.

[9] Sherlock M, O'Sullivan E, Agha A, et al. The incidence and pathophysiology of hyponatraemia after subarachnoid haemorrhage. Clin Endocrinol (Oxf) 2006;64(3):250-4. 
[10] Parandoush S, Abbasi Z, Mohammadi NS, et al. Hyponatremia in traumatic brain injury patients: Syndrome of Inappropriate Antidiuretic Hormone (SIADH) versus Cerebral Salt Wasting Syndrome (CSWS). J Inj Viol Res 2012;4(3 Suppl 1):17.

[11] Kolmodin L, Sekhon MS, Henderson WR, et al. Hypernatremia in patients with severe traumatic brain injury: a systematic review. Annals of Intensive Care 2013;3(1):35.
[12] Schaefer M, Link J, Hannemann L, et al. Excessive hypokalemia and hyperkalemia following head injury. Intensive Care Med 1995;21(3):235-7. 